\title{
Manejo quirúrgico de estadíos primarios en cáncer de labio: Reporte de caso
}

\author{
Surgical management of primary stages in lip cancer: case report
}

Grajeda-Cruz JA ${ }^{1 a}$, Gálvez-Juárez $\mathrm{Y}^{2 \mathrm{a}}$, Nava-Cruz $\mathrm{EM}^{2 \mathrm{~b}}$, Benitez-Cárdenas $\mathrm{OA}^{1 \mathrm{c}}$, Noyola-Frías MA ${ }^{3 \mathrm{~d}}$.

\section{RESUMEN}

El carcinoma oral de células escamosas es considerado la neoplasia maligna más común en labio, y una de las principales causas se debe a la radiación solar, entre otros factores. La forma más común de presentación clínica se manifiesta como lesión ulcerada, endofítica o exofítica que aumenta progresivamente e infiltra por continuidad las zonas adyacentes, con alto riesgo de metástasis regional a ganglios linfáticos o a órganos distantes, de modo que es de suma importancia su atención en estadios primarios para mejorar el pronóstico de la enfermedad, así como también conocer el óptimo manejo, siendo la cirugía el tratamiento de elección. Dicho lo anterior, se presenta un caso de cáncer en labio superior, y su protocolo de atención desde la valoración hasta el manejo, preservando la función y estética del paciente.

Palabras Clave: Cáncer oral; Células escamosas; Tratamiento; Labio. (Fuente: DeCS BIREME)

\section{ABSTRACT}

Oral squamous cell carcinoma is considered the most common malignancy in the laboratory, and one of the main causes is due to solar radiation, among other factors. The most common form of clinical presentation is manifested as an ulcerated, endophytic or exophytic lesion that progressively increases and infiltration by continuity in the adjacent areas, with a high risk of regional metastasis to lymph nodes or distant organs, so their attention is of utmost importance in primary stages to improve the prognosis of the disease, as well as to know the treatment, with surgery being the treatment of choice. That said, a case of cancer is presented in a superior laboratory, and its protocol of care from assessment to management, preserving the function and aesthetics of the patient.

Key Words: Oral cancer; Squamous cells; Treatment; Lip. (Source: MeSH NLM)

1 Especialidad Cirugía Oral y Maxilofacial. Hospital Central "Dr. Ignacio Morones Prieto" de San Luis Potosí. México

2 Especialidad Dermatología. Hospital Central "Dr. Ignacio Morones Prieto" de San Luis Potosí. México.

3 Servicio de Cirugía Oral y Maxilofacial. Hospital Central "Dr. Ignacio Morones Prieto" de San Luis Potosí. México.

\section{a Residente}

b Adjunto

c Coordinador Académico

d Jefe

\section{Correspondencia:}

Jonathan Alexis Grajeda Cruz

Matehuala \#170, Col Lomas, CP 78219, San Luis Potosí, SLP. Teléfono: 3315188260

Correo electrónico: jonathangrajedar5@gmail.com

Citar como: Grajeda-Cruz JA, Gálvez-Juárez Y, Nava-Cruz EM, Benitez-Cárdenas OA, Noyola-Frías MA. Manejo quirúrgico de estadios primarios en cáncer de labio: Reporte de caso. KIRU. 2020;17(4): 209-212. https://doi.org/10.24265/kiru.2020.v17n4.5 


\section{INTRODUCCIÓN}

El carcinoma oral de células escamosas (COCE) es la onceava neoplasia maligna más común en el mundo. A pesar de la tendencia mundial con ligera disminución en la incidencia de casos, se muestra variabilidad de acuerdo con la ubicación geográfica en la que se diagnostica. ${ }^{1}$ Según los datos de GLOBOCAN estimó en el 2018 un total de 18.1 millones de casos nuevos y 9.6 millones de muertes, de los cuales por sitio en cavidad oral se dieron 354,864 de nuevos casos y 177,384 de muertos, en Norteamérica se estima 45.780 casos nuevos de cáncer oral y faringe, en Latinoamérica la situación es muy similar teniendo las incidencias más altas se reportaron en Argentina, sur de Brasil y Uruguay. ${ }^{2}$ Los factores de riesgo más comunes del cáncer oral es el uso de tabaco y alcohol de los cuales se sabe que actúan de forma sinérgica cuando existe el consumo de ambos, así también la exposición a luz solar, consumo de nuez de betel, dieta pobre en frutas y verduras, entre otros.

El objetivo del manejo es el control a largo plazo del cáncer con preservación de la función y estética de la cavidad oral. La cirugía continúa siendo el principal tratamiento inicial, sin embargo, la escisión quirúrgica puede afectar significativamente la función oral y calidad de vida. En tumores en estadios primarios del labio se debe conocer las alteraciones que el tratamiento quirúrgico puede causar sin afectar la estética y funcionalidad. ${ }^{3}$ Así también se puede tener en cuenta la radioterapia como tratamiento inicial o coadyuvante, no obstante, las secuelas a largo plazo de la radioterapia en labio incluyen atrofia de la piel y musculatura subyacente, xerostomía, mucositis, osteoradionecrosis, entre otros. Es importante saber que el drenaje linfático en labio es rico, aunque la diseminación es poco frecuente en los tumores en estadio temprano. Por lo tanto, el tratamiento quirúrgico en los ganglios linfáticos regionales, solo se realiza en tumores primarios avanzados. ${ }^{4}$

El tratamiento quirúrgico en los primeros estadios del cáncer en labio se clasifica según el método para restaurar el defecto causado por la resección del tumor, por lo que se dividen en tres grupos: 1. Resección y cierre primario, 2. Resección y reconstrucción con colgajo local y 3 . Resección con transferencia de tejido libre. Todo esto en virtud del tamaño, la ubicación del tumor y los requerimientos de reconstrucción ya que defectos más grandes requieren reconstrucción compleja con transferencia de tejido libre. ${ }^{5}$

El objetivo de este trabajo es presentar un caso de COCE en labio estadio temprano, localizado en labio superior y evidenciar la importancia de un diagnóstico precoz, así como su tratamiento adecuado.

\section{Reporte de caso}

Se presenta un paciente masculino de 44 años de edad, sin antecedentes crónico-degenerativos, albañil de profesión, exposición a luz solar crónica sin uso de bloqueador solar en región facial, es referido de Centro de salud en Santa María del Rio al servicio de especialidad Dermatológica en coordinación con la especialidad de Cirugía Maxilofacial, del Hospital Central Dr. Ignacio Morones Prieto, S.L.P., por presentar nódulo exofítico ovoideo en labio superior lado derecho, de bordes bien delimitados, superficie café violácea, con úlcera central cubierta por costra hemática, la lesión es no dolorosa y presenta un diámetro de 20 mm de un mes de evolución. Figura 1.

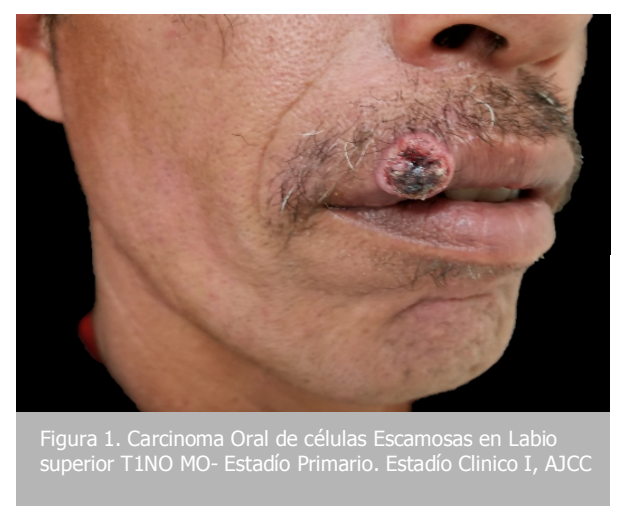

Se realiza una Biopsia Incisional por punch de $5 \mathrm{~mm}$ de diámetro, y se envía al servicio de Patología para estudio Histopatológico donde se evidencia el diagnóstico microscópico: Carcinoma oral de células escamosas, moderadamente diferenciado.

Se analiza el caso de forma multidisciplinaria y de acuerdo con el estadio clínico de enfermedad (EC I), tamaño y localización del tumor T1, N0, M0, así como la extensión necesaria de resección, se sugieren tres posibilidades de tratamiento quirúrgico: a) Resección en $\mathrm{V}$ con cierre primario sin comprometer la comisura, b) Resección en $\mathrm{V}$ con reconstrucción más colgajo de rotación Abbe-Estlander y c) Resección rectangular más reconstrucción con reparación unilateral de triangulo nasogeniano. Figura 2.

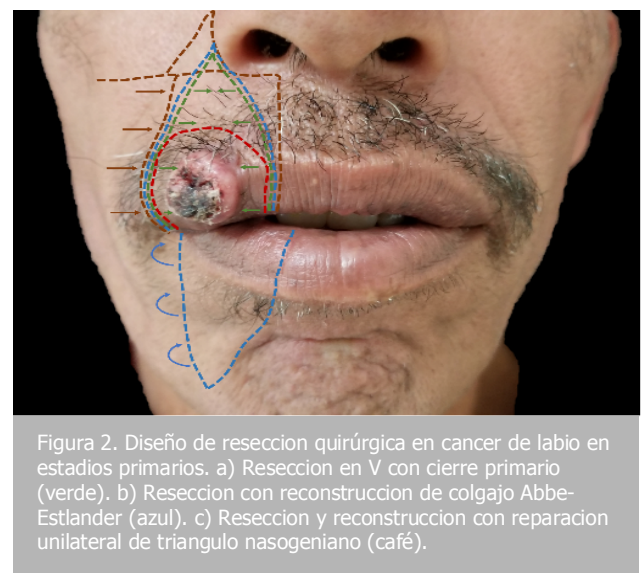


Contemplando las posibilidades quirúrgicas se tomó la decisión en base a determinantes importantes en el análisis Facial, paciente leptosomico de acuerdo con la clasificación de Kretschmer, clase II de angle, biotipo de piel Mixto, escala de Glogau Tipo II, flacidez moderada de tercio medio facial. Lo cual nos llevó a establecer como tratamiento quirúrgico definitivo la resección total del tumor en $\mathrm{V}+$ cierre primario, esto con la finalidad de preservar la función y estética facial en el postoperatorio, estableciendo los cuidados y compromiso vascular de la región, teniendo en cuenta la eliminación del tumor con márgenes libres de seguridad de $10 \mathrm{~mm}$. (Figura 3)

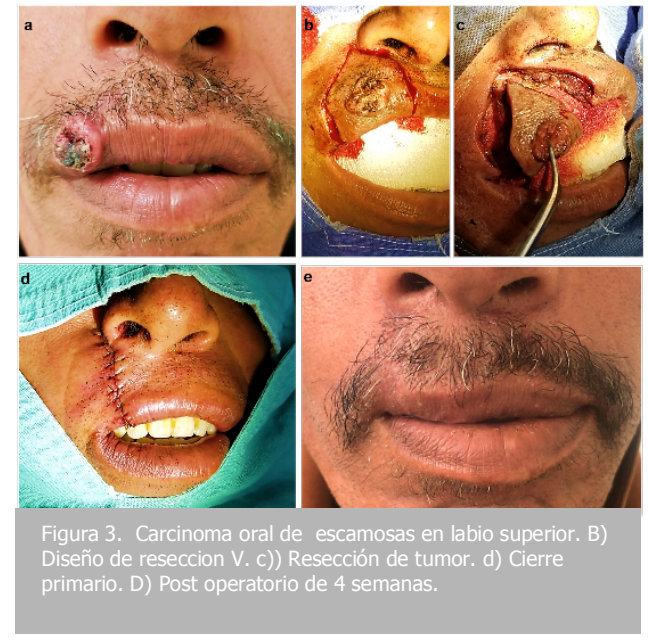

El análisis histopatológico reportó: carcinoma de células escamosas moderadamente diferenciado convencional GII, unifocal ulcerado, con infiltración hasta musculo y tejidos blandos, bordes empujantes, sin invasión linfovascular ni perineural presente, márgenes libres de células neoplásicas, de acuerdo con la clasificación de AJCC, 2018. (Figura 4) Actualmente se encuentra en periodo de vigilancia, sin recurrencia de la enfermedad y con adecuada preservación de la función y estética facial.

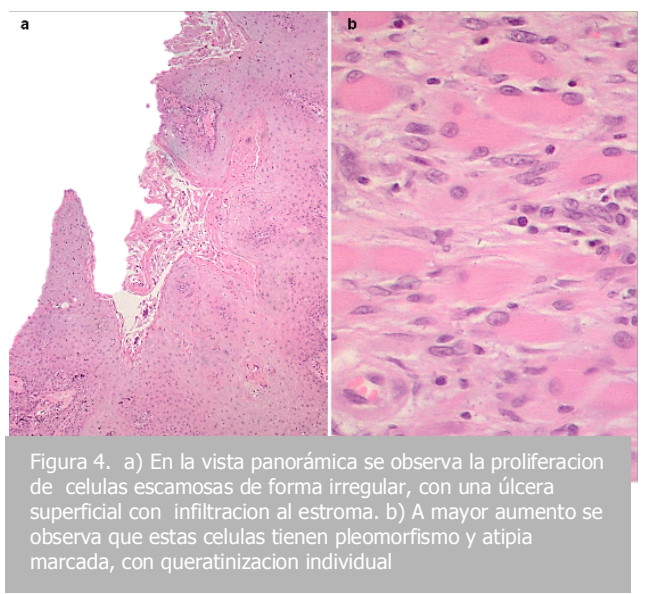

\section{Discusión}

El labio es una estructura compleja debido a la presencia de múltiples tejidos como: piel, mucosa, glándulas salivales menores, músculos y estructuras neurovasculares. Por lo cual pueden presentarse una gran variedad de neoplasias en este sitio, sin embargo, la neoplasia más frecuente en labio es el carcinoma oral de células escamosas. Suele asociarse con áreas geográficas con climas soleados, en personas que trabajan bajo la luz solar de manera directa $o$ en contacto con sustancias químicas carcinógenas, existen factores desencadenantes extrínsecos e intrínsecos. Dentro de los extrínsecos podemos destacar el papel que juegan las radiaciones ionizantes, como los rayos $X$, grenz $y$ gamma, el virus del papiloma humano, diversas sustancias químicas como hidrocarburos (hollín, alquitrán de hulla y aceites cortantes), el consumo de alcohol y tabaco, así como a infecciones recurrentes del virus del herpes simple humano, factores extrínsecos: algunas genodermatosis como el xeroderma pigmentoso, el albinismo, la inmunosupresión.

El sitio de la lesión primaria con mayor frecuencia es en labio inferior hasta en un $89 \%$, el $7 \%$ surgen en el labio superior, y el $4 \%$ surgen en la comisura. ${ }^{5}$ La razón por la cual se tiene mayor porcentaje en labio inferior está asociado a la exposición de luz solar ya que este será el sitio que recibirá directamente radiación solar, sin embargo se sabe que en pacientes con Clase II de Angle, donde el tercio medio facial tiene mayor exposición a la radiación solar siendo el labio superior el que tiene mayor probabilidad de ser afectado por la exposición solar, ${ }^{5}$ es por ello que se asoció las condiciones anatómicas con la actividad laborar de nuestro paciente y los pocos cuidados ante la exposición solar, el sitio anatómico donde se presentó este caso.

La AJCC menciona la presentación clínica en el cáncer oral se manifiesta como: úlcera, tumor endofítico o tumor exofítico principalmente, como resultado del crecimiento del tumor, razón por la cual al ingreso del paciente y evaluar la lesión existente en labio superior se decidió tomar biopsia de forma mediata para realizar un tratamiento oportuno, ${ }^{7}$ sin embargo se debe tener en cuenta los posibles diagnósticos diferenciales como: el carcinoma basocelular, el queratoacantoma, el melanoma, queratosis actínica tipo queratótico e hiperplásico, tumores derivados de los anexos de la piel, dermatofibroma, y otros tumores fusocelulares menos frecuentes como el fibroxantoma atípico. ${ }^{8}$

No existe un método ideal para la reconstrucción de los labios. Cuando la lesión ocupa más del 35\% del labio, para un adecuado cierre es necesaria una reconstrucción con colgajos, teniendo en cuenta el 
margen de seguridad de 3-9 mm. , forma de presentación, tamaño del tumor, localización, grado de diferenciación, tipo histológico, grado histológico, recurrencia, tratamientos previos con radioterapia $u$ otros. ${ }^{9}$ Por las dimensiones del tumor y condiciones del paciente se decidió realizar la resección del tumor en $\mathrm{V}$ con cierre primario, teniendo especial cuidado en: la preservación de ramas del nervio facial para prevenir una disfunción del labio, la conservación de los vasos arteriales y de elemental importancia, realizar una adecuada resección del tumor contemplando los márgenes para evitar la persistencia de la enfermedad, asi también evitando un procedimiento más agresivo que comprometiera mayores estructuras.

En la recurrencia del tumor se aconseja realizar la cirugía de Mohs., la radioterapia está indicada en localizaciones en que se necesite la preservación del órgano y la función o como adyuvante posterior al tratamiento quirúrgico en especial en resecciones con márgenes positivos a células neoplásicas. Se debe dar seguimiento estrecho durante cinco años, para detectar una posible recurrencia o metástasis de la enfermedad la cual se puede presentar entre el 10 $30 \%$ de los casos, o bien el desarrollo de un carcinoma en otra localización anatómica. ${ }^{10}$

\section{Conclusiones}

Es de elemental importancia la inclusión de especialistas en el área para la atención integral en estadios primarios de cáncer oral, un factor determinante en la reducción de la morbilidad y mortalidad. La cirugía continúa siendo la piedra angular en el tratamiento del cáncer primario, en tumores de labio se pueden manejar con cierre primario o injerto de piel, se tiene que tomar en cuenta siempre la mejor opción funcional y estética del caso ya que el éxito del procedimiento quirúrgico será basado en el conocimiento desde el comportamiento de la enfermedad hasta las alteraciones que se presentaran según el tratamiento realizado, siempre teniendo en cuenta el mejor resultado para el paciente.

Contribuciones de autoría: JGC, YGJ, EMNC,OABC y MANF diseñaron el estudio, recopilaron y analizaron la información, redactaron y aprobaron el artículo.

Fuente de financiamiento: El estudio fue financiado por los autores.

Conflicto de intereses: Los autores declararon no tener conflictos de interés.

\section{BIBLIOGRAFÍA}

1. Global Incidence and risk factors of oral cancer. Ghantous Y, Abu Elnaaj L. 10, India : Harefuah, 2017, Vol. Oct 156. 645-649.

2. Global Cancer Statistics 2018: GLOBOCAN Estimates of Incidence and Mortality Worldwide for 36 Cancers in 185 Countries. Freddie Bray, BSc, MSc, PhD, Jacques Ferlay, ME y Isabelle Soerjomataram, MD, MSc, PhD. 394-424, Lyon, France: Ca Cancer Journal Clinic, 2018, Vol. 68.

3. Functional considerations in oral cavity. Patrik Pipkorna, Kelsey Rosenquist, Joseph Zenga. 00, St Louis, Missouri, USA : Curr Opin Otolaryngol Head Neck Surg, 2018, Vol. 26.

4. Cancer, American Joint Committee on. AJCC Cancer Staging Form Supplement. Chicago, USA : American College of Surgeons, 2018.

5. Wong, Jatin Shah Snehal Patel Bhuvanesh Singh Richard. Head and Neck Surgery and Oncology. New York USA : Elsevier, 2019. 5th.

6. Unusual Growth of Upper Lip Squamous. Stojanovic S, Jovanovic M, Vuckovic N. 3, Iran: Crescent Med J, 2015, Vol. 13

7. Distribucion y riesgo geograficodelcancer oral. Grajeda-Cruz J.A., López-Verdín S. , VázquezBojórquez C. , Soto-Ávila J.J. . 1, Guadalajara, Mexico: Revista Medica-Cientifica de la Secretaria deSalud Jalisco, 2019, Vol. 1.

8. Comportamiento Epidemiologico del Programa de Deteccion del Cancer Bucal. Sánchez EZ, Casanova SK, Hidalgo TY,. 10, Puerto Padre : Zoilo, 2014, Vol. 39.

9. Lower Lip of Recontruction. Ernesto Carmona Fernández, Alexei Perez Hernandez, Aurora Velazquez Martinez, Maria del Carmen Ginebra Rodriguez. 2, Pinar del Rio: Revista Ciencias Medicas, 2017, Vol. 21.

10. Cancer Epidemology. Medical Exposures, Including Hormone Therapy. Friis, S Kesminiene A, Espina C, Auvienen A, Straif K. 1, European Code : CCM, 2016, Vol. 39.

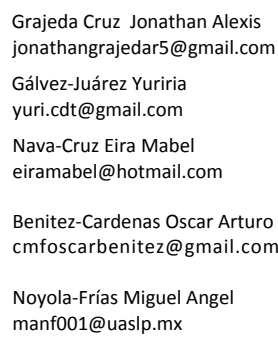

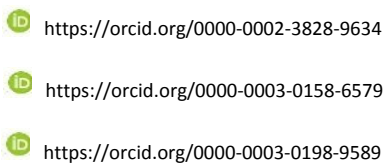

